\title{
Optimization of Tasks using Scheduling Algorithms in Cloud Computing
}

\author{
G. Sumathi, S.Rajesh
}

\begin{abstract}
Thinking about the creating usage of disseminated processing and the prerequisite for perfect use of assets in the cloud, and respect for customers that pay for organizations they use subject to their pay as-you-go premise, there should be a snappier course for customers to reduce the customer's holding up time and undertaking's holding up time. The guideline explanation behind this paper is to give a perfect estimation using the upsides of the two customary Min-Min and Max-Min calculations. The other point that follows in this calculation (PBQACOMMP) is the priority of the undertakings. There are huge amounts of booking calculations on the planet today, yet the priority given to the undertakings has been overlooked and ignored in numerous calculations. In this calculation, priority is right off the bat decided for errands subject to a prioritization calculation, and a short time later using the center number to pick which one of the Min-Min or Max-Min calculations is to be used. It should be seen that pursued by PBQACOMMP calculations, its holding up time is lower than relationships of the differentiated calculations and is showed up with be better than the comparable calculations.
\end{abstract}

Keywords - Ideal scheduling algorithm, planning algorithm, cloud planning algorithm, Min-Min algorithm, Max-Min algorithm, cloud computing

\section{INTRODUCTION}

Because of the increasing development of capability and processing innovation, and the success of the net, the rateviability of resource getting geared up and accessibility, this innovation has brought approximately the improvement of some other processing model known as disbursed computing. In which property and applications are discharged after the purchaser's utilization and is imparted to special customers. Reality be informed, allotted computing makes an adaptable domain for giving sources[1] and relying on expansive computer systems. Dispensed computing has some of assets on hand for clients in distinct routes, for instance, virtualization, net conventions, and so forth[2]. Cloud computing conceals its specialised points of hobby from the point of view of customers, and placing layers among those diffused elements and customers[3]. Task Scheduling is critical in distributed computing [19] because it straightforwardly impacts a frameworks load and execution. A compelling challenge Scheduling approach requires not simply meeting the patron's desires but further improving the efficiency of the whole framework. The ant colony set of rules has been applied for a giant range of making plans issues, undertaking promising outcomes [20][25] .In allotted computing, resources and obligations are altogether extraordinary. For instance, some obligations have an attraction for the CPU, even as some require greater

Revised Manuscript Received on April 12, 2019.

G. Sumathi, Kalasalingam Institute of Technology, Krishnankoil 626126, Tamilnadu, India.

S.Rajesh, Associate Prof., Department of IT, Mepco Schlenk Engg College, Sivakasi - 626005, Tamilnadu, India. garage. The expenses of numerous property range. As goals be, the charges of responsibilities are additionally specific. Consequently, it is useful to mirror project charges inside the event that we recall the call for distinction of the responsibilities for the belongings in element. On the way to cope with this issue, we propose a aid cost technique. This version can replicate the requests of the duties for the assets in element. Likewise, this paper proposes a multi-goal optimization scheduling version that takes into document the 2 imperatives of execution and price range price. Subsequently, it tackles the multi-target optimization scheduling trouble using the ant colony set of guidelines, which has an superb preferred standpoint in tending to this combinatorial optimization problem.But, it is easy to fall into a nearby most suitable. Even as using the ant colony set of policies, consequently, this paper proposes a licensed ant colony set of rules that might estimate further to adjust the super of the answer with the intention of avoid falling into that local most excellent. The number one goal of this scheduling technique is overall performance and rate variety price; due to the fact it's miles primarily based on the ant colony optimization set of rules, it is named PBQACOMMP. We suggest three models for challenge optimization: there are resource fee model, multi-intention optimization-scheduling model and qualified ant colony optimization model (it's going to solves the trouble that the particular ant colony algorithm that continuously falls into the nearby gold trendy) interest Scheduling is a way in which a selected aid is appointed to an challenge at a predetermined time. The number one motivation at the back of the planning is to increase useful aid talent and its vital format is to lower keeping up instances. The planning of Jobs in allocated computing is a standout many of the maximum difficult jobs. "A green scheduling can extend the framework's proficiency." [2] In 2nd part of this text we keep in mind approximately the associated work. In 1/3 part we make clear The project Optimization set of rules dependent on the MAX-MIN, MIN-MIN and Priorities (PBQACOMMP) and its centers. The correlation amongst PBQACOMMP and distinct algorithms is in fourth detail. Furthermore, stop is spoken to in fifth component.

II. Related paintings In a calculation, parameters of task duration and priority responsibilities had been considered Inside the occasion that the calculation precedence to run relying on the period of the responsibilities, it to begin with orchestrates the jobs prepared by the use of period of duties, at that component figures the not unusual period of the responsibilities, and in a while ascertains the distinction 
inside the period of each hobby with the imply. This calculation chooses Jobs from the centre and gives credit score to each jobs, and lines for execution. In the event which you priority to keep strolling as in keeping with the concern, as consistent with the precedence given by way of using the purchaser to the hobby, credit is mounted and the roles are stored strolling based totally totally on their legitimacy[5] . In a Dynamic useful useful resource Scheduling set of rules, a jitter calculation is proposed wherein the calculation of the advent time of a call for for awesome jobs is considered as a fundamental concept. With reference to making plans Jobs, the interest that has more first rate project accessible (execution time) is before the whole thing set. This calculation distributes property to clients right now in making ready[6]. A calculation is proposed for organizing jobs, which might be orchestrated within the primary level as indicated with the resource of the season of access. In the 2 nd step, the roles are prepared with the aid of the due date and set inside the organized line. The interest that takes precedence is carried out earlier than. In the event that when the line kind, every other precedence activity is added to the road, it'll be positioned toward the start of the road and the road is probably reordered. In reality, they make use of the EDF (Earliest cut-off date First) calculation. [7] the focal point is on reducing the time associated with scheduling overall performance, inclusive of the response time, maximum notable span and of entirety time [24]_[27]. For example, reference [6] proposes a brilliant heuristic set of rules the precept goal of that is the gold preferred span. A propelled max-min planning calculation is proposed for allotted computing. Or, in different words inside the propelled Max-min calculation. In the first area, it acquires a normal runtime from each single on hand responsibilities, at that factor chooses the nearest run-time separation to the ordinary range. Every so often the best hobby is just too large and it causes framework lopsidedness. This method diminishes Makespan and performs load balancing over the Max-min technique[8] . Within the system arranging of calculation, PPIA (priority based absolutely universal performance advanced set of rules) is usually recommended that considers the concern of the customer. Clients who regularly employ cloud structures can come across better company. Glaringly, the best precedence is given to everyday client obligations. Unique jobs are ordinary precedence jobs. The Meta work set ought to be separated into gatherings for critical Jobs and normal priority jobs. In Contrasted with the Min-min calculation, Makepan decreases it and expands the use charge of property[9] .

An ever developing wide kind of appears into highlight on multi-goal optimization. As an example, the multi-target enhancement consists of the consummation time, the necessities of QoS, strength usage, monetary price and the framework execution. The investigates [14] , [15] bear in thoughts the due date ensures. Wherein the paper [14] proposes an errand making plans to make sure due date with the resource of enhancing resource utilization, and the paper [21] proposes a way with the useful resource of maintaining apart assets and financial plan to restrict the consummation time of assignments and make strides beneficial resource usage. This approach considers the reputation of those sources. Severa precise examinations actually streamline the task without thinking about the recognition of the aid [19], likewise targeting power with the resource of assessing the property. The paper [20] unites VMs to spare power via the use of the subterranean insect kingdom framework. A multiinput multi-yield complaint control of dynamic beneficial useful resource reserving calculation was proposed [27] to ensure ideal viability under time barriers. This calculation considers the errand execution time, rate, and usage of sources (CPU, memory). In the one-of-a-kind calculation, the 3 calculations RR (round Robin), SJF (Shortest procedure First), and precedence are utilized, with all processes this is in priority strains. The precedence move is low, medium to excessive. With respect to RR calculation, an inexpensive quantum is given to all tactics. Thusly, low priority bureaucracy are less tedious than beginning procedures, and moderate priority paperwork have a lot much less time than excessive priority forms. Given the RR calculation, every interest has a duration reduce for the use of the processor. Quantum time ought not to be low as it makes an expansive range of switches. The concern calculation likewise places the CPU on pinnacle priority jobs, and if the two duties have a similar priority, at that point uses the primary Come First service (FCFS) calculation [10] .

With admire to imperfections of the two time-venerated Min-Min and Max-Min calculations, it's far proposed to triumph over those algorithmic errors, first earlier than managing the and so forth network (anticipated Time to Compute) making use of Min-Max standardization to scale the qualities inside the variety characterized. Within the wake of normalizing, the new estimations of the NETC are grid someplace in the variety of zero and 1, or, in other words to pick Jobs. Here, the attractive digital machine for each hobby is characterized due to the fact the virtual device, which units apart the lowest opportunity to play out this jobs [11].

In a pre-allotment device for arranging responsibilities in dispensed computing, some other p.C. (Pre-assignment Ant Colony Optimization) calculation has been supplied depending on ACO (Ant Colony Optimization). This calculation joins an more effective ACO calculation and layout measure free of charge venture planning. The proposed method and calculation performed well in the simulation surroundings. The ones examinations screen that $\%$. Can enhance making plans execution [12] .

With the issue of enhancing the SJF making plans calculation, an algorithmic distributed computing calculation is proposed to accumulate the splendid outcome of planning, deliver down execution time, and diminishing Make span. The primary modified SJF precept belief is to address jobs in growing request relying on the length of duties and ascertaining the ordinary of the combination duration of the jobs. In this paper, the SJF work making plans calculation is changed to accomplish the deliberate jobs with the base Makespan. It focuses not certainly throughout the fulfilment time of the duties, but additionally 
on the very last touch of all subjects considered. We exhibited with observational proof that MSJF is superior to SJF and FCFS. Trial effects confirmed that MSJF (modified Shortest manner First) became greater efficient in enhancing reaction time and Makespan contrasted with SJF and FCFS [13] .

The calculation grow to be given priority aware Longest challenge First (PA-LJF). Inside the ones customers, they ship matters thru the net interface. The jobs are with an assistant accepting the call for. The client who sent the interest is confirmed if the hobby sent from him is despatched to the priority line, generally the client can be known as the aggressor and his interest might not be finished. Each this form of calculations has beneficial conditions and inconveniences. For this reason, as in keeping with the customer's priority or selection, the making plans calculation is selected. After the cease of the hobby, the calculation's execution is limited, least run-time, excessive effectiveness, response time, information transfer functionality applied and consumer fulfilment is checked [14] .

In development calculations, enhancement may be utilized to enhance planning parameters, for instance, price, vitality, beneficial aid use, turnaround time, and runtime. An algorithmic resource became proposed to lessen Makespan and increment useful useful resource profitability. On this calculation, it offers making plans to an expansive sort of free jobs to check out the execution of the Makespan and CPU parameters depending at the particle development integration and the Max-Min calculation. Thusly, this calculation joins the upsides of the 2 calculations with exquisite precision. For that reason, it reveals an appropriate arrangement earlier than the normal one. Exploratory results display the superb execution of this calculation with unique calculations [15] .

The RASA (useful resource aware Scheduling set of guidelines) calculation is characterized so if the quantity of assets on hand is unusual, the Min-min approach is hooked up to allocate the primary pastime, usually the Max-min device applies. The rest of the jobs are allotted to at least one in every of techniques to their person assets. For example, if the number one challenge allocated to a Minmin technique, the subsequent interest is dictated thru the machine of Max-min. The time unpredictability of the RASA calculation is same to $2 \mathrm{O}(\mathrm{MN})$, where $\mathrm{M}$ speaks to the quantity of assets and $\mathrm{N}$ speaks to the quantity of jobs, or, in distinct phrases complexity of the calculation Maxmin and Min-min [16] .

In the extra user preference-based totally smart Scheduling set of rules (E-UPISA) considers the precedence powerfully. For the first time, they offer uniformity the whole way and as indicated via the use of the crucial priority that clients frequently utilize. A device that won't be applied for quite some time will reduce their priority. Trial effects show that E - UPISA lessens the retaining up time, rotational time, and response time for the coveted strategies, and diminishes in popular framework execution contrasted with everyday programming calculations. In this paper, consumer inclinations are applied due to the fact the purpose for arranging. The proposed calculation uses dynamic priority standards [17].

\section{PROPOSED ALGORITHM}

The usage of the upsides of the two extensive Min-Min, Max-Min computations and a sincerely ideal rely has been introduced to reduce the retaining on schedule for assignments. The general limit of the PBQACOMMP computation is a line of employments, each venture has:

Identity: range of every venture

Exe_time: Runtime

Pri: priority enrolled with the aid of the use of the purchaser.

Credit score score, pri_fac, total_credit: the ones components are set via the priority paintings.

Wt: Saves the maintain on schedule for each undertaking.

From the outset we ship occupations to the priority paintings, with the aid of then we were given the yield, next take into account alternatives the underlying 11 undertakings and picks the one that has the most diminished execution precedence (the greatest extensive range) and parcels it by using 2, to get the suggest quantity. By then it locate the quantity of extra noteworthy and humbler errands of the recommend wide range if the amount of greater diminutive undertakings outperforms the quantity of employments which can be greater by way of the use of then use the Min-Min figuring and if the amount of greater assignments outperforms the quantity of smaller assignments, use the Max-Min computation. After the satisfaction of the underlying 11 errands, they will do in like manner for severa Jobs. This technique is completed to the final touch of the street.

The dataset is placed within the show, with the aid of then the bunch is despatched to the begin paintings. This bunch is multi-dimensional and has the traits recorded already.

\section{A. The System Model}

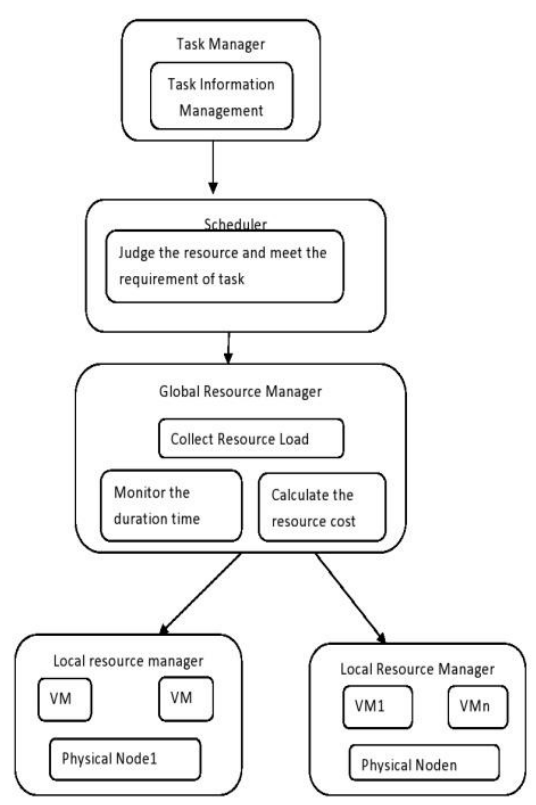

Fig 1: System model architecture

Published By: Blue Eyes Intelligence Engineering \& Sciences Publication 
The project solicitations are acknowledges and oversees with the aid of the undertaking director which might be ship with the aid of the patron and this statistics are deliver to the scheduler. The community property are monitors and oversee by means of the nearby asset manager and moreover checking close by virtual property to get their CPU and reminiscence load information, and after that send this to the worldwide belongings director. The data are accrued and refreshed by means of way of the worldwide property administrator this is despatched from nearby belongings supervisor. The network property supervisor additionally monitors the span time of project running on the assets and sends it to the worldwide belongings administrator. Utilizing this facts from the supply version, the asset charge is decided via the global administrator.

The middle phase is the Scheduler . The use of the streamlining approach, the scheduler assigning undertakings to property. From the errand manager and international belongings director, it purchases that undertaking and assets facts, Then it makes a desire about the property $\mathrm{Rj}$ meets the need of the project Ti.It incorporates the actual prerequisite, price and due date.

Atlast, the property $\mathrm{Rj}$ is assigned to the challenge Ti with the aid of the scheduler.

\section{B. Start characteristic}

The rule restrict of the estimation is the begin paintings. In all honesty, this limit makes sense of which computation is executed. To start with, the undertakings are despatched to the priority functionality to decide the challenge precedence reliant at the computation within the priority art work. Through then the roles display is gotten due to the fact the yield of the concern figuring and positioned in the 11 bunch of obligations. By using and by means of way of, the various eleven duties, select the only that has the maximum faded priority, and a while later parcel by means of 2 to get a mean range. ( 1 is most surprising precedence and 99 is the least precedence) Now, if the amount of assignments which have a higher priority is greater conspicuous than the undertakings that have a lower priority, the Min-Min be counted is used and if the amount of errands that have a low precedence isn't always as a good deal as the errands which have an increasingly more critical precedence, the Max-Min figuring is used. Along those traces, you could take tendencies of each normal Min-Min and Max-Min figurings.

Across the end, the protective up time is figured, which, given the effects were given and the examinations finished display that the matter quantity has an appealing execution.

\section{Want feature}

In this restrict, from the outset, it finds the most shocking ( $\max )$ and least (min) priority, and after that their regular (avg) is decided. This precedence is given by way of the usage of the customer to all of the employments.

Through the usage of and by way of manner of traits (from regard 1 to deal with 4) are discovered out as seeks after:

Regard 1 is identical to the setting apart max via 5

Regard 2 is identical to the setting apart max through 4

Regard 3 is equal to the mixture of price 2 and cost 1

Regard 4 is same to the mixture of fee 2 and cost three
By using and through for all of the errands in the line, the avg need to be diminished from their runtime and positioned in the TLD variable.

By means of then, if the TLD is certainly lower than cost 1 , its credit score is similar to 5 .

If our TLD is among charge 2 and value 1, its credit rating is 4 .

In case our TLD is among fee 3 and fee 2, its credit score score is 3 .

In case our TLD is among price four and price 3 , its credit score is .

If the TLD is extra essential than fee 4, its credit score is proportional to 1 .

Thru figuring out and consigning credit score score for every project, make feel of pri_fac play the going with advances:

For all the occupations within the pri_fac line, every assignment is similar to the concern of a comparable venture divided with the aid of manner of 10 .

By way of and by means of manner of it is an exceptional chance to play out the last period of this restrict and sign on the entire credit score score. For all duties inside the precedence line, their regard is same to the aftereffect of the credit score rating increment in pri_fac.

Yield of this limit is an series of priority employments. That is a comparative computation determined in [5], it's carried out right here

\section{Min function}

On this functionality, the occupations are in a comparative solicitation from the humblest motion to the greatest interest reliant on their remaining precedence. It must be visible that as verified through the begin art work, the road incorporates 11 undertaking. As a depend of reality, this restrict is the comparable because the Min-Min estimation. Anyways, on this limit, unlike the Min-Min figuring, the road is deliberate with the useful resource of the concern of the obligations, no longer established on their runtime.

Next to orchestrating the road, the occupations are deliberate from the maximum vital precedence (which has a greater unobtrusive quantity than the rest, for example, task 1 has precedence 1 , so this venture has the maximum vital priority) to the least priority, and protective up time of this line is the yield that has been ship to the begin work.

\section{E. Max feature}

In this capability, the errands are in the line from the pleasant motion to the humblest motion situation to their last priority. It need to be visible that as verified by means of the begin paintings, the street carries eleven task. Absolutely, this capacity is the similar as the traditional Max-Min estimation, except for that during this capability, now not inside the least likes the Max-Min figuring, the road is engineered via the concern of the obligations, now not mounted on their runtime.

Next to organizing the road, the occupations are coordinated from minimum precedence to the most 
dumbfounding priority and the preserving on agenda for this line as yield is sent to the begin art work.

IV. Calculation usual overall performance effects

Duplicate trials had been primarily based utilising Cloudsim to verify the presentation of PBQACOMMP

\section{A. Trials and parameter Setup \& RESULTS}

The examinations took a gander on the reserving approach of this paper with the first subterranean insect province calculation, the installation heuristic recollect MinMin estimation [23], [24] and FCFS arranging. The primary subterranean insect province estimation have become so to talk used to format without thinking about spending necessities. The Min-Min figuring has remarkable feature on making plans time and bases on improving final touch instances. Our tests made a server ranch using Cloudsim three.Zero. There had been two hundred hosts and 20 digital machines on every host. The parameter association of VMs in the server dwelling house is seemed table 1 . The parameter association of endeavors is confirmed up in desk 2.

Table 1.The factor setup of VMs in Data Center

\begin{tabular}{|c|c|}
\hline Factor & Rate \\
\hline CPU Computing ability & 2660MIPS,3990 MIPS \\
\hline RAM & $8192 \mathrm{MB}$ \\
\hline Band Width & $200 \mathrm{M} / \mathrm{s}$ \\
\hline Storage & $20 \mathrm{G}$ \\
\hline
\end{tabular}

Table 2.The factor setup of task in data center

\begin{tabular}{|c|c|}
\hline Factor & Value \\
\hline Length(CPU) & {$[800,2000] \mathrm{MIPS}$} \\
\hline File Size & {$[400,2000] \mathrm{MB}$} \\
\hline Output Size(Memory) & {$[40,80] \mathrm{MB}$} \\
\hline The number of Tasks & {$[200,1200]$} \\
\hline
\end{tabular}

Also, the quantity of ants in the ant colony algorithm and in the original ant colony algorithm is 20 in the tests.

\section{B. Analysis Measurements}

The examinations used four execution appraisal hints: make range, that's the overall time everything taken into consideration moreover, is used to assess reserving execution; fee of client, which became differing due to unique making plans systems and property; due date infringement rate, this is the facts effect of QoS due to the fact that a wonderful solid shape calls for complaint to check its execution; the fourth marker is asset utilization.

\section{First examination}

Round there, using the dataset got from [12] , the examination of the PBQACOMMP computation with the percent estimation is executed from [12] and the Min-Min and Max-Min counts, and the consequences are showed up in desk three and decide 2.

As showed up in desk 3 and determine 2, the execution of the PBQACOMMP computation is higher than different for all intents and functions indistinguishable estimations and has a lower Make duration.
Table 3. Comparison of PBQACOMMPwith PACO and Min-Min and Max-Min

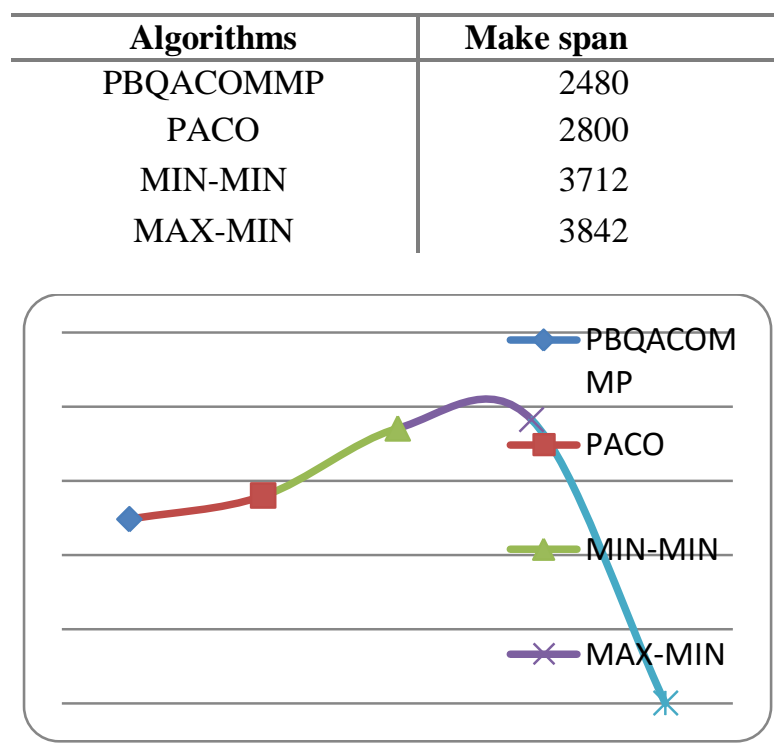

Fig 4. Comparison of PBQACOMMP with PACO and Min-Min and Max-Min

\section{Second correlation}

In this portion, with the dataset got from [18], we differentiated the PBQACOMMP computation and the Ataskmin-maxmin count from [18], which the results are showed up in Table 4 moreover, Fig. 3.

As showed up in Table 4 and Fig.3, the execution of the PBQACOMMP estimation is better than the Ataskminmaxmin computation and has a lower Make range.

\section{Table 4.Comparison of PBQACOMMP with taskmin-} maxmin

\begin{tabular}{l|l}
\hline Algorithms & Make span \\
\hline TASKMIN-MAXMIN & 16469.94 \\
PBQACOMMP & 12863.36
\end{tabular}

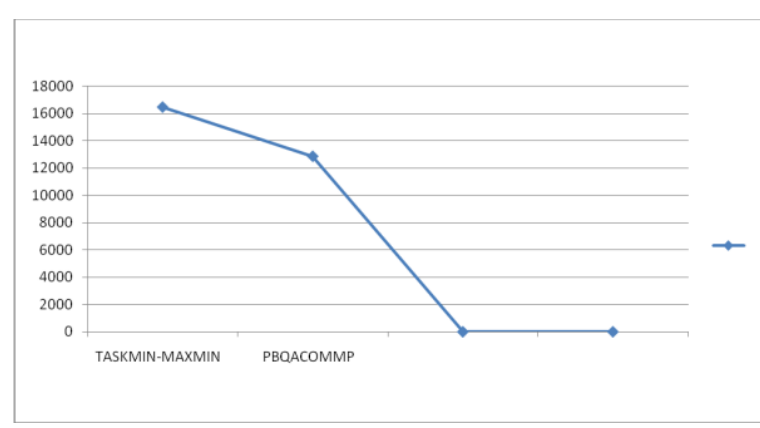

Fig 5. Comparison of PBQACOMMP with taskminmaxmin

\section{E. Third examination}

In this area, by the dataset from the paper [13], we contrasted the PBQACOMMP calculation and the MSJF 
calculation from [13], the aftereffects of which are appeared in Table5 and Fig6.

Table 5 .Comparison of PBQACOMMP with MSJF

\begin{tabular}{c|r}
\hline Algorithms & Make span \\
\hline PBQACOMMP & 10130.36 \\
MSJF & 21014
\end{tabular}

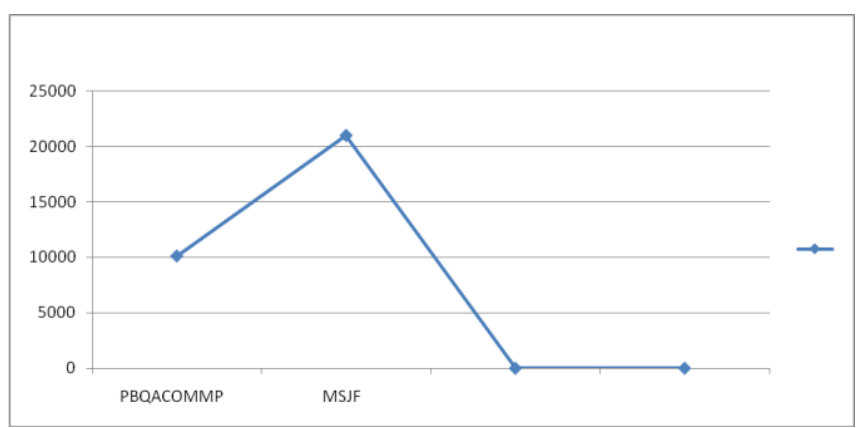

Fig 6.Comparison of PBQACOMMP with MSJF

As appeared in Table 5 and Fig. 6, the execution of the PBQACOMMP calculation is superior to the MSJF calculation and is less Make span.

\section{F. Fourth correlation}

In this segment, by dataset acquired from [15] the PBQACOMMP calculation contrasted and the PBCOPSO calculation and Max-Min_PSO from [15], the outcomes are appeared in Table4 and Fig4.

$\begin{array}{lc}\text { Table 6. Compare PBQACOMMP with PBCOPSO and } \\ \text { Max-Min_PSO } \\ \text { Algorithms } & \text { Make span } \\ \text { PBQACOMMP } & 572.54 \\ \text { PBCOPSO } & 698.64 \\ \text { MAX-MIN_PSO } & 664.48\end{array}$

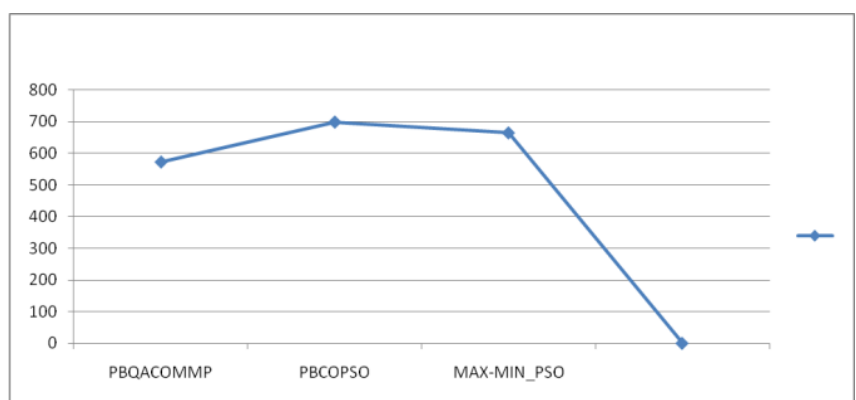

Fig IV. Compare PBQACOMMP with PBCOPSO and Max-Min_PSO

As appeared in Table 4 and Figure 4, the execution of the PBQACOMMP calculation is superior to PBCOPSO and Max-Min_PSO calculations and has a lower Make span.

A multi-target improvement planning technique (PBACO) Stepped forward towards becoming proposed depending upon the subterranean insect state calculation. In the primary region, the PBACO helpful asset fee rendition may be linked to symbolize the undertaking aspect hobby for resources. This version responds the affiliation among precious asset fees and way charges. Except, a multi-target progression display come to be proposed, in angle at the model. Its essential goal turned into to modernize the reserving of execution and consumer fees. Thirdly, a showed subterranean insect country count number turn out to be proposed to comprehend the development difficulty. Inside the occasion which you need to maintain up the subterranean insect settlement rely from falling into an adjacent impeccable sport plan, this system used the execution and spending prerequisite talents to evaluate the prices and supply contribution on the character of the course of movement. It via then adjusted the concept of the relationship as proven via the results of the appraisal and evaluation. Exploratory consequences exhibit that the PBACO method has a stunning excellent potential in wording of makespan. Positively, even within the maximum surprisingly lousy state of affairs, PBACO is more or less proportional to Min-Min computation, which has variables of interest as for time. PBACO will increase practically fifty six. $6 \%$ as for the FCFS count. The PBACO methodologies are by means of the by way of reducing facet to some thing other than what's anticipated almost same structures with recognize to uncommon estimations, for instance, fee, the infringement fee of due date and beneficial asset use, showing the reasonability of PBACO.

III. Conclusion This paper, using two normal Min-Min and Max-Min figurings, an a success making arrangements computation (PBQACOMMP) moved closer to becoming proposed to diminish the take a seat tight time and Make range for occupations within the CLOUD registering system. Within the wake of selecting the want for all employments through using the assessing count, it picks the underlying 11 undertakings; at that thing finds out the imply amount, and as tested by means of making use of the measure of occupations whose precedence is a widespread first-rate arrangement than the endorse wide assortment, it alternatives one of the same old computations Min-Min or Max-Min.

The yield of the agonies shows that the PBQACOMMP rely is better than stand-out estimations. In like way, for the reason that the overhead of the 2 conventional figurings focused on is low and the PBQACOMMP depend utilizes the upsides of the 2 computations made affiliation with over, the proposed estimation overhead is without a doubt too low.

\section{REFERENCES}

1. Meriam, E. What's greater outstanding, N. Tabbane, "a evaluation on Cloud Computing Scheduling Algorithms", worldwide Summit on laptop and information length, 2016.

2. Wadhonkar, A. What is greater, D. Theng. "a framework on unique making preparations estimations in administered processing", global gathering on Advances in electrical, Electronics, statistics, file and Bio-Informatics (AEEICB16), 2016

3. Jain, S. Moreover, A.Ok. Saxena, "A Survey of Load Balancing problems in Cloud condition", 5th global display on machine Modeling and headway in studies styles, IEEE, 2016.

Published By: Blue Eyes Intelligence Engineering \& Sciences Publication 
4. Zamanian. SH, Rostaee. R, "making preparations for Cloud Computing", 1/3 countrywide display on laptop Engineering and facts age manipulate, Shahid Beheshti college.2016

5. Solymani. Z, Ghavami. B, " the new matter of arranging and shelling out sources within the cloud condition", general amassing on computer Engineering and information duration, 2016

6. Ajay Thomas S. What's extra distinguished, Santhiya. C, "Dynamic beneficial asset Scheduling the use of eliminate Time set of concepts in Cloud environment", second basic collecting On Computing and Communications innovation (ICCCT'17). IEEE, 2017.

7. Gupta, G., et al., "A Simulation of priority basically based Earliest cut-off date First Scheduling for Cloud Computing device", First regular show on Networks and easy Computing, IEEE, 2014.

8. Santhosh B., Manjaiah D. H, and L.Pandma Suresh, "A Survey of differing Scheduling Algorithms in Cloud situation", typical collecting on rising Technological propensities [ICETT]. IEEE, 2016.

9. Amalarethinam, D.I.G. What's extra, S.Kavitha, "need based by using and big execution propelled calculation for Metaadventure Scheduling in Cloud environment", second regular meeting On Computing and Communications technology(ICCCT'17), IEEE, 2017

10. Parekh, H.B. What is greater prominent, S. Chaudhari, "greater grounded spherical Robin CPU Scheduling set of requirements", global display on global traits in sign Processing, insights Computing and record, IEEE, 2016.

11. Gupta R., et al., "A ground-breaking Multi-objective mission Scheduling set of requirements using Min-Max Normalization in Cloud Computing", 2d global assembly on linked and Theoretical Computing and verbal change age (iCATccT), IEEE, 2016.

12. Lin, R. Additionally, Q. Li, "task Scheduling calculation essentially depending on Pre-Allocation approach in Cloud Computing", IEEE global show on Cloud Computing and sizable facts evaluation. 2016.

13. Alworafi, M.A., et al., "An progressed SJF Scheduling set of concepts in Cloud Computing environment", global accumulating on electric, Electronics, correspondence, computing device and Optimization methods, IEEE, 2016.

14. Kumar, M. What's extra prominent, S.C. Sharma, "Priorityt cognizant Longest motion First (PA-LJF) calculation for usage of the asset in cloud environment", international show on Computing for Sustainable international development, IEEE 2016.

15. Jain, A. Similarly, R. Kumari, "An powerful asset utilization primarily based absolutely blanketed undertaking Scheduling calculation", fourth global gathering on sign Processing and fused Networks (SPIN), IEEE, 2017.

16. Parsa, S. Except, R. Entezari-Maleki, "RASA: any other venture Scheduling set of requirements in Grid environment". Universal did Sciences magazine 7 (amazing inconvenience of pc and IT), 2009.

17. Kamal, R., et al., "up to date customer inclination basically based totally clever Scheduling calculation (E-UPISA)", protests of the twenty $1 / 3$ international show on Automation and Computing, college of Huddersfield. 2017.

18. B, Santhosh. Also, Manjaiah D. H, "A Hybrid AvgTask-Min and Max-Min calculation For Scheduling responsibilities In Cloud Computing", in common display on control,Instrumentation, file and Computational improvements (ICCICCT), IEEE, 2015.

19. C. Zhu, V. C. M. Leung, X. Hu, L. Shu, and L. T. Yang, "An audit of key troubles that situation the attainability of cell disbursed computing," in Proc. IEEE Int. Conf. Digital, Phys., Soc. Comput. (CPSCom), Aug. 2013, pp. 769_776.

20. Y. Chen, A. Zhang, and Z. Tan, "Complexity and estimation of single machine reserving with an administrator non- accessibility duration to diminish combination of sum time," Inf. Sci., vol. 25, no. 1, pp. 150_163, Dec. 2013.

21. R. Van nook Bossche, okay. Vanmechelen, and J. Broeckhove, "cost ef_cient making plans heuristics for reduce-off date confined extremely good tasks at hand on go breed mists," in Proc. IEEE third Int. Conf. Cloud Comput. Technol. Sci. (CloudCom), Nov./Dec. 2011, pp. 320_327.

22. S. Very well. Garg, R. Buyya, and H. J. Siegel, "Time and fee trade off control ment for planning parallel bundles on application lattices," predetermination Generat. Comput. Syst., vol. 26, no. Eight, pp. 1344_1355, Oct. 2010.

23. H. Kellerer and V. A. Strusevich, "completely polynomial wager plans for a symmetric quadratic backpack issue and its making plans bundles," Algorithmica, vol. Fifty seven, no. 4, pp. 769_795, Aug. 2010.

24. C.- W. Tsai, W.- C. Huang, M.- H. Chiang, M.- C. Chiang, and C.- S. Yang, "A hyper-heuristic making plans calculation for cloud," IEEE Trans. Cloud Comput., vol. 2, no. 2, pp 236_250, Apr./Jun. 2014.

25. Z. Tang, L. Jiang, J. Zhou, adequate. Li, and okay. Li, "A selfadaptivescheduling set of pointers for reduce begin time," predetermination Generat. Comput. Syst., vols. 43_44, no. Three, pp. 51_60, Feb. 2015.

26. B. Tripathy, S. Sprint, and S. Very well. Padhy, "Dynamic project booking the utilization of a coordinated neural system," J. Parallel Distrib. Comput., vol. 75, no. Five, pp. 101_106, Jan. 2015.

27. H. Topcuoglu, S. Hariri, and M.- Y. Wu, "by means of and huge execution amazing and espresso unpredictability task booking for heterogeneous figuring," IEEE Trans. Parallel Distrib. Syst., vol. Thirteen, no. Three, pp. 260_274, Mar 2002.

28. Y.Wang andW. Shi, "accounts pushed reserving calculations for clumps of MapReduce employments in heterogeneous mists," IEEE Trans. Cloud Comput., vol. 2, no. 3, pp. 306_319, Jul./Sep. 2014.

29. M. A. Rodriguez and R. Buyya, "due date primarily based asset provisioningand planning calculation for scienti_c work_ows on mists," IEEE Trans. Cloud Comput., vol. 2, no. 2, pp. 222_235, Apr./Jun. 2014.

30. M. Hu and B.Veeravalli, "Dynamic making plans of half of breed ongoing commitments on bunches," IEEE Trans. Comput., vol. 33, no. 12, pp. 2988_2997, Dec. 2015.

31. All right. $\mathrm{Li}, \mathrm{X}$. Tang, and all right. $\mathrm{Li}$, "manage ef_cient stochastic enterprise booking on heterogeneous processing structures," IEEE Trans. Parallel Distrib. Syst., vol. 25, no. Eleven, pp. 2867_2876, Nov. 2014. 